a Universidade Federal de São Carlos, Departamento de Engenharia de Produção, Campus de Sorocaba, CEP 18052-780, Sorocaba-SP, Brasil. b Universidade Federal de São Carlos, Programa de Pós-Graduação em Ciência dos Materiais, Campus de Sorocaba, CEP 18052-780, Sorocaba-SP, Brasil. - Universidade Federal de São Carlos, Programa de Pós-Graduação em Engenharia de Produção, Campus de Sorocaba, CEP 18052-780, Sorocaba-SP, Brasil.

\section{*E-mail: jane@ufscar.br}

Recebido em: 2 de Abril de 2021

Aceito em: 19 de Abril de 2021

Publicado online: 29 de Julho de 2021

\section{Resíduos Sólidos Urbanos como Fonte de Energia Renovável no Brasil: Panorama Atual e Perspectivas Futuras}

\author{
Municipal Solid Waste as Source of Renewable Energy in Brazil: Current \\ Overview and Future Perspectives
}

\author{
Karen de Souza do Prado, ${ }^{a, b}$ Christian Cuello Barrios, ${ }^{c}$ Anderson Pires Fernandes, ${ }^{c}$ Jane Maria \\ Faulstich de Paiva $a, b, c, *$ (1)
}

\begin{abstract}
With the increase in the Brazilian population and the demand for energy in large cities, the use of municipal solid waste (MSW) as a renewable energy source arises as a promising alternative. However, there is still low interest in its use due to the lack of information compiled about its potential. For this reason, this work aimed to present the current status of the MSW energy recovery in Brazil and to analyze the works published in this area in the last five years, in order to understand what their future perspectives are and to stimulate their use in the country. Through an updated bibliographic survey, it was observed that at least $65 \%$ of MSW have potential for energy reuse. The wet waste includes biomass (51.7\%) and polymeric waste $(13.5 \%)$. The analyzed studies revealed great concern with the management of MSW, mainly in the southeastern region of the country, and that the energy reuse of MSW through the techniques of incineration, biodigestion and gasification shows great economic and environmental potential. Despite the significant amount of available MSW, greater attention must be paid to this renewable energy source in order to make it a representative part in the Brazilian energy matrix.
\end{abstract}

Keywords: Municipal solid waste; biomass; polymeric waste

\section{Introdução}

Com o aumento da população brasileira, que supera 212 milhões de habitantes, ${ }^{1}$ é também crescente a demanda por energia, principalmente nas grandes cidades. De acordo com dados da Empresa de Pesquisa Energética, enquanto o consumo de energia elétrica em 2018 foi de aproximadamente $424 \mathrm{TWh}$, é estimado que o consumo total de eletricidade no Brasil aumente em $75 \%$ até 2026, podendo chegar a $744 \mathrm{TWh} .{ }^{2,3}$ Neste contexto, torna-se necessário o estímulo ao uso de fontes de energia renováveis, que possam suprir a demanda energética do país com o menor impacto ambiental possível.

Enquanto fontes renováveis como solar, eólica, geotérmica, hidráulica e biomassa correspondem a apenas 14,0 \% da matriz energética mundial, as fontes renováveis são responsáveis por suprir $42,9 \%$ da energia usada no Brasil. ${ }^{4}$ A participação três vezes maior de fontes renováveis na matriz energética brasileira quando comparada à mundial deve-se, principalmente, à grande disponibilidade de biomassa, notadamente derivados da cana, lenha e carvão vegetal.

Outra fonte de energia renovável com grande potencial de uso no Brasil são os resíduos sólidos urbanos (RSU). Em 2018, a média de geração de RSU por habitante no Brasil foi de $380 \mathrm{~kg}$, totalizando 79 milhões de toneladas de resíduos gerados. ${ }^{5}$ Deste total, $92 \%$ foram coletados e 59,5\% destes foram destinados a aterros sanitários (equivalente a 47 milhões de toneladas), enquanto mais 29 milhões de toneladas de RSU foram descartadas em locais inadequados, trazendo sérios riscos para o meio ambiente e para a saúde das pessoas. ${ }^{5}$ Como a taxa de reaproveitamento dos RSU ainda é muito baixa (em torno de 10\%, de acordo com dados do Programa das Nações Unidas para o Meio Ambiente), ${ }^{6}$ o uso dos RSU para geração de energia surge como uma alternativa promissora pois grande parte destes resíduos possui alto poder calorífico, ${ }^{7}$ sendo estimulada por planos governamentais e normativas nacionais como a Política Nacional de Resíduos Sólidos. ${ }^{8}$

Entretanto, ainda existe um grande desinteresse no uso dos RSU como fonte de energia renovável, principalmente devido à falta de informações compiladas sobre suas potencialidades. ${ }^{9}$ Este trabalho teve como objetivos apresentar o panorama atual do reaproveitamento de RSU para a geração de energia no Brasil e fazer uma análise dos trabalhos publicados na área nos 
últimos cinco anos, visando compreender quais são as perspectivas futuras desta fonte de energia renovável e estimular seu uso no país.

\section{Metodologia}

Os dados apresentados neste trabalho foram obtidos por meio de um levantamento bibliográfico realizado em sites de órgãos oficiais relacionados à geração, ao descarte e ao reaproveitamento energético dos RSU, da biomassa e de resíduos poliméricos, como a Agência Nacional de Energia Elétrica, a Empresa de Pesquisa Energética, o Programa das Nações Unidas para o Meio Ambiente, a Associação Brasileira de Empresas de Limpeza Pública e Resíduos Especiais, a Associação Brasileira da Indústria do Plástico e o Ministério de Minas e Energia, tendo como base o Plano Nacional de Energia 2030. ${ }^{9}$

Também foi realizada uma busca bibliográfica na base Web of Science no dia 02 de março de 2020 com os tópicos "municipal solid waste" AND "energy recov*" AND "Brazill, que retornou em 39 artigos publicados nos últimos cinco anos. Estes artigos foram revisados para adequação ao tema deste trabalho, e a exclusão de trabalhos não relacionados ao reaproveitamento energético dos RSU no Brasil resultou em 32 artigos.

Para análise qualitativa dos artigos selecionados, foi usado o software IRaMuTeQ (Interface de R pour les Analyses Multidimensionnelles de Textes et the Questionnaires), v. 0.7 alpha 2, com funcionalidades do software estatístico $\mathrm{R}$ 3.5.0. O corpus original foi preparado em Word a partir dos resumos dos artigos selecionados, resultando em 32 textos separados por linhas de comando compreendendo uma variável (*****Artigo_1, *****Artigo_2, etc.) e organizados em um único arquivo. Após revisão da ortografia e gramática, o arquivo foi salvo em bloco de notas usando a codificação de caracteres no padrão UTF-8. A partir do corpus, os segmentos de texto foram analisados por meio do software IRaMuTeQ usando as ferramentas de estatística, de classificação hierárquica descendente (CHD) em função dos respectivos vocabulários e da frequência de ocorrência no texto, e da análise de similitude. Substantivos e verbos foram considerados como formas ativas para as análises. Os resultados obtidos foram correlacionados com os dados encontrados nos órgãos oficiais.

\section{Resultados e Discussão}

\subsection{Panorama atual da recuperação energética dos RSU no Brasil}

De acordo com dados recentes da gravimetria dos RSU coletados no Brasil, a fração úmida, da qual faz parte a biomassa, corresponde à maior fração dos resíduos coletados $(51,7 \%)$, seguida por outros tipos de resíduos $(16,7 \%)$ e pelos resíduos poliméricos $(13,5 \%) .{ }^{10}$ Portanto, tem-se que pelo menos $65 \%$ dos RSU coletados possuem potencial para reaproveitamento energético, considerando resíduos na forma de biomassa e de resíduos poliméricos, o que corresponde a aproximadamente 51 milhões de toneladas de resíduos. No entanto, a maior parte destes resíduos é disposta em aterros, ${ }^{5,11}$ e não existem ainda usinas de recuperação energética dos RSU em larga escala no país.

\subsection{Recuperação energética da biomassa}

A biomassa presente nos RSU é classificada como rejeito urbano e é composta majoritariamente por resíduos alimentícios e material de origem orgânica proveniente de feiras, comércios, jardins ou áreas verdes. ${ }^{9,12}$ Ela pode ser reaproveitada por meio de diferentes métodos para obtenção de importantes subprodutos, como fertilizantes, combustíveis e energia.

O uso da biomassa dos RSU para recuperação energética é interessante, particularmente para energia elétrica, pois se trata de uma das fontes renováveis com maiores possibilidades em termos de natureza, tecnologias de conversão e produtos. ${ }^{9}$ Dentre as tecnologias de conversão estão processos termoquímicos e bioquímicos, sendo a combustão direta, a gaseificação pela via termoquímica e a digestão anaeróbia as mais usadas para este tipo de rejeito. ${ }^{9}$ Um dos principais produtos obtidos a partir do reaproveitamento energético da biomassa dos RSU é o biogás produzido nos aterros sanitários por via biológica, que pode ser usado para uso doméstico ou industrial, ou ainda ser convertido em energia elétrica, térmica ou em combustíveis. ${ }^{9,12}$ Sendo formado majoritariamente por metano (60 a $80 \%$ ), o poder calorífico do biogás varia em torno de 5000 a $7000 \mathrm{kcal} / \mathrm{m}^{3} .{ }^{13}$

Apesar do grande potencial de uso, existe ainda um grande desinteresse no reaproveitamento energético da biomassa proveniente dos RSU no Brasil. ${ }^{9}$ Isto está associado, principalmente, à dificuldade da separação da biomassa em sua fonte de geração, além dos custos logísticos com o transporte destes resíduos até os centros de tratamento, que geralmente são distantes de suas fontes. ${ }^{12}$ Estas dificuldades limitam estimativas mais precisas sobre a disponibilidade da biomassa proveniente dos RSU, fazendo com que a maioria dos estudos nacionais para reaproveitamento de biomassa utilize resíduos agrícolas, silviculturais ou agroindustriais, como derivados da cana-deaçúcar. Apesar de constituir mais da metade da composição gravimétrica dos RSU, a biomassa proveniente de rejeito urbano ainda não é considerada representativa para ser integrada ao sistema elétrico do país, o que faz com que grande parte destes resíduos ainda seja descartada em aterros..$^{9,11}$

\subsection{Recuperação energética dos resíduos poliméricos}

Em 2018, apenas 16,9 \% dos resíduos poliméricos coletados no Brasil foram destinados a cooperativas de 
reciclagem, ${ }^{5}$ o que significa que quase 67 mil toneladas de resíduos poliméricos foram descartadas em aterros ou em locais indevidos. Nas cooperativas de reciclagem, o resíduo polimérico geralmente é separado, limpo, seco e reprocessado visando à obtenção de um novo produto, em um processo denominado reciclagem mecânica. ${ }^{14}$ Entretanto, esta metodologia de reciclagem somente pode ser aplicada para polímeros termoplásticos, que são capazes de amolecer e fundir com o aumento da temperatura. Para os demais polímeros, chamados termorrígidos (ou termofixos), e nos casos em que o reuso do resíduo polimérico não é prático nem econômico devido à sua quantidade ou contaminação, outras metodologias de reciclagem são possíveis, como as reciclagens química e energética. ${ }^{15,16}$

$\mathrm{Na}$ reciclagem energética, o conteúdo de energia presente nas ligações covalentes é recuperado por meio de processos de incineração, pirólise ou gaseificação, podendo ser reaproveitado na forma de calor, vapor e eletricidade. . $^{16,17}$ A recuperação energética de $1 \mathrm{~kg}$ de resíduo polimérico equivale à queima de $1 \mathrm{~L}$ de óleo combustível, e contribui com cerca de $30 \%$ da energia obtida na recuperação energética dos RSU. ${ }^{16}$

Embora seja a metodologia de reciclagem que mais cresceu no mundo nos últimos anos, sendo usada em larga escala na Europa e em países como os Estados Unidos e o Japão, ainda não existe no Brasil uma usina de reciclagem energética com capacidade suficiente para suprir a demanda das cidades.

\subsection{Análise dos estudos recentes e perspectivas futuras}

Dentre os 32 artigos selecionados, os estudos sobre recuperação energética dos RSU no Brasil concentraramse majoritariamente na região sudeste do país, onde $65 \%$ dos estudos foram realizados; sendo o estado de São Paulo responsável por mais da metade destes trabalhos. Foram também encontrados estudos realizados nas regiões sul e central do país.
A análise estatística revelou que as dez palavras mais usadas pelos autores foram: 1) waste (143 ocorrências), 2) energy (75), 3) management (52), 4) landfill (47), 5) municipal (47), 6) Brazil (43), 7) generation (42), 8) system (34), 9) electricity (33) e 10) recovery (33). Este resultado indica a ênfase dos estudos na gestão e no reaproveitamento energético dos RSU gerados no Brasil, que em sua maioria são descartados em aterros e possuem grande potencial para geração de energia.

A classificação hierárquica descendente dos segmentos de texto é apresentada na Figura 1.

Observa-se que os segmentos de texto foram agrupados em três classes de palavras: a classe 1 com $34 \%$ das ocorrências, referente aos problemas e desafios relacionados à gestão e ao descarte dos RSU no Brasil; e as classes 2 e 3, ambas derivadas da classe 1 , com 33,3 e $32,7 \%$ das ocorrências respectivamente. Enquanto a classe 2 engloba palavras relacionadas à recuperação energética dos RSU, como uma estratégia nacional de gestão dos resíduos descartados nos aterros com grande potencial de retorno ambiental e econômico, a classe 3 agrupa as principais tecnologias de conversão utilizadas atualmente para recuperação energética dos RSU, que são a incineração, a biodigestão anaeróbia e a gaseificação, que são as mesmas usadas majoritariamente para biomassa. Estas tecnologias foram mencionadas respectivamente em 44, 25 e 12,5\% dos artigos selecionados.

A Figura 2 mostra o grafo obtido por meio da análise de similitude.

Na Figura 2 pode ser observada a relação entre os diferentes temas abordados nos artigos referentes à recuperação energética dos RSU no Brasil nos últimos cinco anos. Analogamente às demais análises apresentadas, observa-se que a geração e gestão dos RSU são temas centrais abordados nos artigos (em cor avermelhada, Figura 2). Destas temáticas derivam-se outras como o potencial de recuperação dos resíduos descartados nos aterros (em cor azul, Figura 2), o uso das rotas de conversão

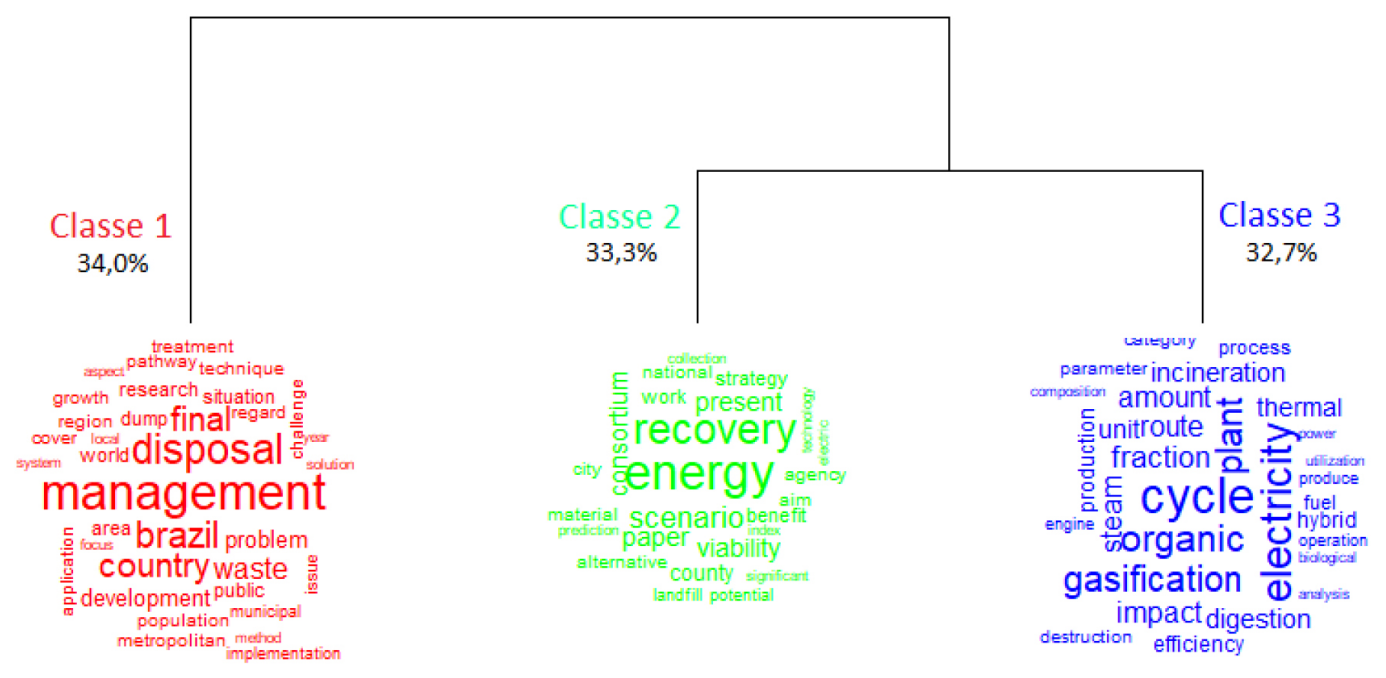

Figura 1. Classificação hierárquica descendente dos segmentos de texto dos artigos selecionados 


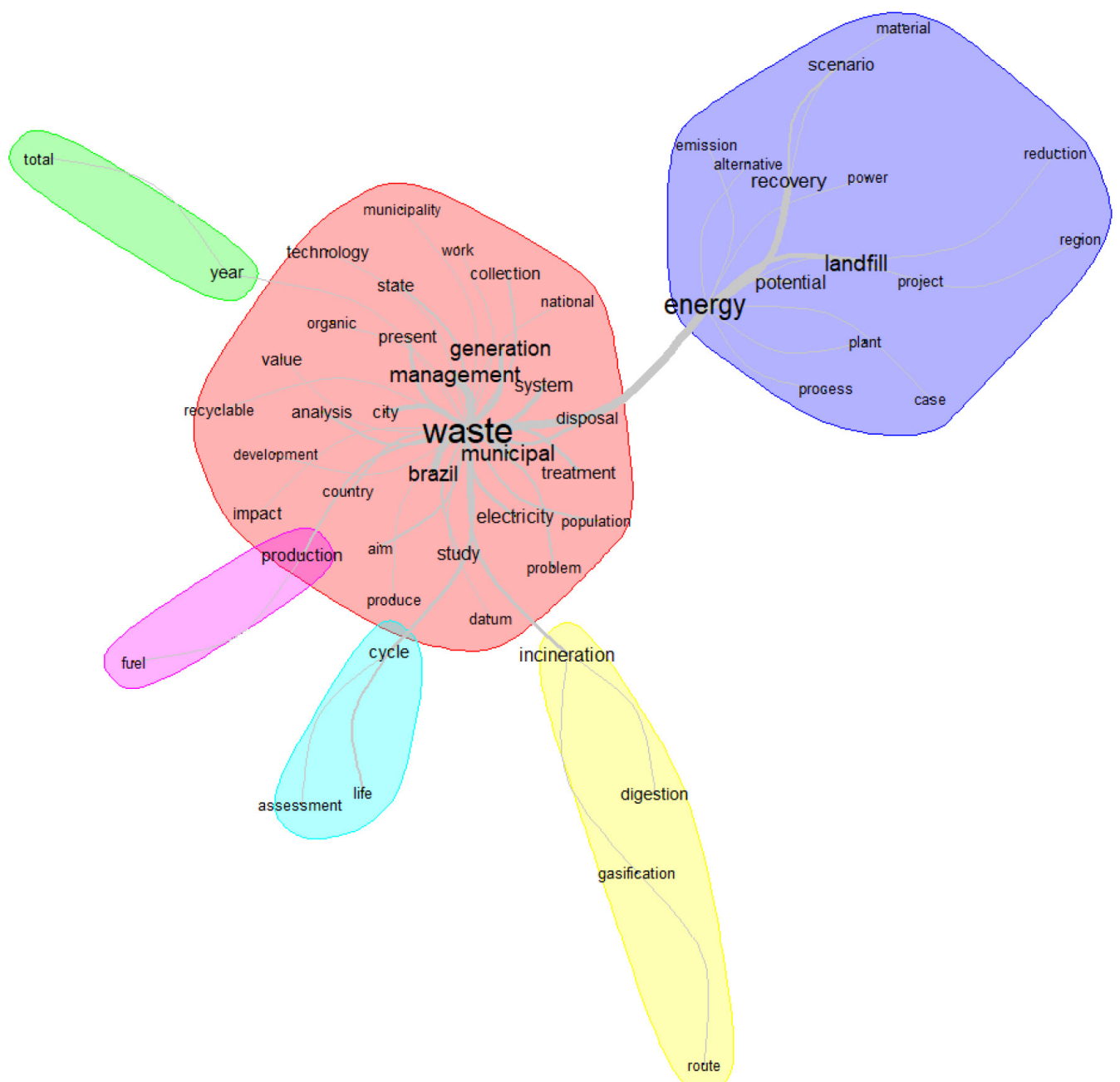

Figura 2. Resultado da análise de similitude dos segmentos de texto dos artigos selecionados

energética por meio da incineração, digestão e gaseificação (em cor amarela), a análise de ciclo de vida destes processos (em cor azul claro), a produção de combustíveis usando seus subprodutos (em cor rosa lilás), e estudos que monitoram a evolução anual do total de RSU produzido, coletado e descartado no Brasil (em cor verde).

Comparando-se a análise qualitativa dos artigos com os dados obtidos de órgãos oficiais, pode-se notar uma estreita relação entre os resultados. Enquanto os estudos recentes têm mostrado a importância da gestão dos RSU e o potencial de sua valorização por meio da recuperação energética, os dados fornecidos pelos órgãos oficiais revelam que a introdução dos RSU na matriz energética brasileira ainda está longe de se tornar realidade, uma vez que ainda não existem usinas de recuperação energética no país capazes de suprir as demandas dos grandes centros urbanos.

Assim, torna-se necessária maior atenção a essa fonte renovável de energia, uma vez que as tecnologias de conversão já utilizadas para biomassa agrícola e agroindustrial como a incineração, a biodigestão e a gaseificação podem também ser usadas para recuperação energética da biomassa presente nos RSU, ${ }^{18,19}$ aproveitando-se também da reciclagem energética dos resíduos poliméricos presentes.

\section{Considerações Finais}

Os resultados deste trabalho revelam que a gestão dos RSU é muito importante para minimizar seu impacto ambiental, bem como para sua valorização. A recuperação energética deste recurso renovável é uma alternativa promissora para o seu redirecionamento dos aterros, porém, ainda pouco explorada no país, apesar da abundância dos RSU. Para que seu reaproveitamento energético seja factível, outras fontes de biomassa devem ser consideradas na matriz energética brasileira e outras tecnologias de conversão precisam ser mais exploradas. Além disso, maiores investimentos precisam ser realizados para viabilizar a utilização de RSU como fonte de energia renovável no Brasil.

\section{Agradecimentos}

Os autores agradecem à CAPES $\left(\mathrm{n}^{\circ}\right.$ processo: 88887.464432/2019-00), Código de Financiamento 001. 


\section{Referências Bibliográficas}

1. Sítio do Instituto Brasileiro de Geografia e Estatística, Projeção da população do Brasil e das Unidades da Federação. Disponível em: <https://www.ibge.gov.br/apps/populacao/projecao/>. Acesso em: 11 março 2021.

2. Sítio da Empresa de Pesquisa Energética, Projeção da demanda de energia elétrica para os próximos 10 anos (20172026). Disponível em: <http://www.epe.gov.br/sites-pt/ publicacoes-dados-abertos/publicacoes/PublicacoesArquivos/ publicacao-245/topico-261/DEA\%20001_2017\%20-\%20 Proje $\%$ C3\%A7\%C3\%B5es\%20da\%20Demanda\%20de\%20 Energia\%20E1\%C3\%A9trica\%202017-2026_VF[1].pdf >. Acesso em: 2 março 2020.

3. Sítio da Empresa de Pesquisa Energética, Consumo Anual de Energia Elétrica por classe (nacional). Disponível em: $<$ https:// www.epe.gov.br/pt/publicacoes-dados-abertos/publicacoes/ Consumo-Anual-de-Energia-Eletrica-por-classe-nacional >. Acesso em: 2 março 2020.

4. Sítio da Empresa de Pesquisa Energética, Matriz Energética e Elétrica. Disponível em: <https://www.epe.gov.br/pt/ abcdenergia/matriz-energetica-e-eletrica $>$. Acesso em: 2 março 2020.

5. Sítio da Associação Brasileira de Empresas de Limpeza Pública e Resíduos Especiais, Panorama dos Resíduos Sólidos no Brasil 2018/2019. Disponível em: <http://abrelpe.org.br/downloadpanorama-2018-2019/>. Acesso em: 2 março 2020.

6. Sítio do United Nations Environment Programme, Waste Management Outlook for Latin America and the Caribbean. Disponível em: <https://wedocs.unep.org/ bitstream/handle/20.500.11822/26448/Residuos LAC EN.pdf?sequence=2\&isAllowed $=\mathrm{y}>$. Acesso em: 3 março 2020.

7. Drudi, K. C.; Drudi, R., Martins, G.; Antonio, G. C.; Leite, J. T. C.; Statistical model for heating value of municipal solid waste in Brazil based on gravimetric composition. Waste Management 2019, 87, 782. [CrossRef]

8. Brasil, Lei $n^{\circ} 12.305$, de 2 de agosto de 2010, Diário Oficial da União, Brasília, seção 1, mar./ago., 2. trim. 2010. Legislação Federal e marginalia. [Link]
9. Brasil, Ministério de Minas e Energia; colaboração Empresa de Pesquisa Energética. Plano Nacional de Energia 2030 - v. 8 - Geração termelétrica a partir da biomassa. Brasília: MME: EPE, 2007. [Link]

10. Sítio do Compromisso Empresarial para a Reciclagem, Review 2019. Disponível em: <https://cempre.org.br/wp-content/ uploads/2020/11/CEMPRE-Review2019.pdf > . Acesso em: 02 março 2020.

11. Alfaia, R. G. S. M.; Costa, A. M.; Campos, J. C.; Municipal solid waste in Brazil: A review. Waste Management \& Research 2017, 35, 1195. [CrossRef] [PubMed]

12. Sítio do United Nations Environment Programme, Organic Waste Management in Latin America: Challenges and Advantages of the Main Treatment Options and Trends. Disponível em: $<$ https:// abrelpe.org.br/pdfs/publicacoes/report onu_eng.pdf $>$. Acesso em: 3 março 2020.

13. Barros, T. G.; Biogás. Disponível em: <https://www. agencia.cnptia.embrapa.br/gestor/agroenergia/arvore/ CONT000fbl23vn102wx5eo0sawqe3qf9d0sy.html>. Acesso em: 3 março 2020.

14. Zanin, M.; Mancini, S. D.; Resíduos Plásticos e Reciclagem: Aspectos gerais e tecnologia, 2a ed., EdUFSCar: São Carlos, 2004.

15. Sítio PlasticsEurope, Recycling and energy recovery. Disponível em: <https://www.plasticseurope.org/en/focus-areas/circulareconomy/zero-plastics-landfill/recycling-and-energy-recovery>. Acesso em: 3 março 2020.

16. Spinacé, M. A. S.; De Paoli, M. A.; A tecnologia da reciclagem de polímeros. Química Nova 2005, 28, 65. [CrossRef]

17. Gonçalves, C. K.; Dissertação de Mestrado, Universidade de São Paulo, 2007. [Link]

18. Medina Jimenez, A. C.; Nordi, G. H.; Palacios Bereche, M. C.; Bereche, R. P.; Gallego, A. G.; Nebra, S. A.; Evaluation of two different alternatives of energy recovery from municipal solid waste in Brazil. Waste Management \& Research 2017, 35, 1137. [CrossRef] [PubMed]

19. Nordi, G. H.; Palacios-Bereche, R.; Gallego, A. G.; Nebra, S. A.; Electricity production from municipal solid waste in Brazil. Waste Management \& Research 2017, 35, 709. [CrossRef] [PubMed] 